\title{
Androgen Receptor Ligand-binding Domain-encoding Plasmid DNA Vaccine MVI-118
}

National Cancer Institute

\section{Source}

National Cancer Institute. Androgen Receptor Ligand-binding Domain-encoding Plasmid DNA Vaccine MVI-118. NCI Thesaurus. Code C121777.

A cancer vaccine containing pTVG4 plasmid DNA encoding the human androgen receptor (AR) ligand-binding domain (LBD) (pTVG-AR), with potential immunostimulating and antineoplastic activities. Upon intradermal administration of AR LBD-encoding plasmid DNA vaccine MVI-118, the plasmid DNA vaccine expresses AR LBD and may stimulate the host immune system to generate a cytotoxic T-lymphocyte (CTL) response against AR LBD-expressing prostate cancer cells. This reduces proliferation of AR-expressing tumor cells. AR, a tumor-associated antigen (TAA) overexpressed in prostate cancer cells, plays a key role in the development and progression of prostate cancer; its expression is correlated with poor prognosis. 